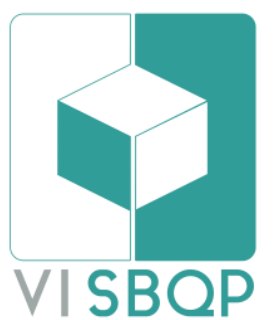

UBERLÂNDIA 2019

\title{
CENTRO DE ATENÇÃO PSICOSSOCIAL: TRANSFORMAÇÃO DA CLÍNICA TRADICIONAL DE MUNDOS ISOLADOS EM LUGAR DA MULTITERRITORIALIDADE
}

\author{
OLIVEIRA, Sarah Gabriela de Carvalho \\ PROAC/UFJF, e-mail: sarah.gabriela20@gmail.com \\ ABDALLA, José Gustavo Francis \\ FAU/PROAC/UFJF, e-mail: gustavofrancis@globo.com
}

\begin{abstract}
RESUMO
A Reforma Psiquiátrica foi estabelecida para outorgar os direitos das pessoas com sofrimento mental e reabilitá-las psicossocialmente. Com a sua formalização legal em 2002, "novos dispositivos e tecnologias de cuidados foram estabelecidos para desconstruir o hospital psiquiátrico em suas bases conceituais e assistenciais e promover a cidadania e a inserção social de pessoas que sofrem com os transtornos mentais" (AMARANTES, 2003, p.7). Esses novos equipamentos, destacando os Centros de Atenção Psicossocial (CAPS), buscam, em meio aos processos assistenciais formais e humanos-ambientais, trabalhar com a lógica da heterogeneidade e a construção social para a re-territorializar seus usuários na cidade. $O$ objetivo do presente artigo é discutir a complexidade da mudança de filosofias e práticas do sistema manicomial para a Reforma Psiquiátrica, e seus reflexos na relação entre usuário, serviço, espaço, cultura, cuidado cognitivo e subjetividade. A investigação tem caráter de pesquisa exploratória, uma vez que há escassos trabalhos acadêmico-científicos no contexto aqui abordado, e utilizará conceitos da Psicologia Ambiental para analisar o ambiente físico, componentes comportamentais das atividades de vida diária e os processos de territorialização dos usuários da rede de Saúde Mental.
\end{abstract}

Palavras-chave: CAPS, Território, Reforma Psiquiátrica.

\begin{abstract}
The Psychiatric Reform was established to concede the rights of people with mental disabilities. The legal formalization happened in 2002: "new devices and care technologies were established to deconstruct the psychiatric hospital and to promote citizenship and the social insertion of people suffering from mental disorders" (AMARANTES, 2003, p.7). These new equipments, highlighting the Centro de Atenção Psicossocial (CAPS), work with the logic of heterogeneity and social construction to re-territorialize their users in the city. The aim of the present article is to discuss the complexity of the change of philosophies and practices of the asylum system for the Psychiatric Reform, and its reflexes in the relation between user, service, space, culture, cognitive care and subjectivity. The research has the character of exploratory research and it will use concepts from Environmental Psychology to analyze the physical environment, behavioral components and the processes of territorialization of the users of the network of Mental Health.
\end{abstract}

Keywords: CAPS, Territory, Psychiatric Reform.

\section{INTRODUÇÃO}

A proposta de reinserção social da nova política de Reforma Psiquiátrica é complexa e engloba aspectos que vão além da desospitalização da população interna dos manicômios. O hospital psiquiátrico, como instituição total, atuava por meio pressupostos totalitários para controlar e docilizar o paciente e torná-lo submisso as regras e ao corpo profissional da instituição

OLIVEIRA, S. G. C.; ABDALLA, J. G. F. Centro de atenção psicossocial: transformação da clínica tradicional de mundos isolados em lugar da multiterritorialidade. In: SIMPÓSIO BRASILEIRO DE QUALIDADE DO PROJETO NO AMBIENTE CONSTRUÍDO, 6., 2019, Uberlândia. Anais... Uberlândia: PPGAU/FAUED/UFU, 2019. p. 1504-1512. DOI https://doi.org/10.14393/sbqp19135. 
(GOFFMAN, 2010, p.17). Os paradigmas que envolvem o tratamento mental ultrapassam o circuito hospitalar e é enraizado na sociedade por meio de estigmas que caracteriza o portador de sofrimento mental de louco, violento e incapaz. A ressocialização, proposta pela Reforma Psiquiátrica, não pode ser apenas um processo que fecha o manicômio, mas tal processo pode e deve refletir e modificar práticas que existem a séculos. Estudos sobre territorialização mostram-se importante neste processo, implicando que o CAPS e os demais equipamentos têm a potencialidade de reconstruir territórios existenciais, englobando interação dinâmica entre cenários sociais do cotidiano, formação de multiterritorialidades e estruturação de um novo lugar social para seus usuários.

\section{REFERENCIAL TEÓRICO}

\subsection{O Hospital Psiquiátrico como Instituição Total}

Goffman (2010) conceitua a instituição total como uma organização que atua com uma estrutura de poder englobante. Possui um sistema rigoroso de normas formais explícitas e um corpo de pessoas aderentes que permite um ritual quotidiano pré-estabelecido imposto por uma hierarquia profissional, distante do usuário, no qual as necessidades humanas e de trabalho são essencialmente manipuladas pela burocracia. Um aspecto significativo destes locais é a usurpação do Eu e ataque direto à moral do sujeito, por uma constante regulação e limitação de atitudes e ações pessoais, intramuros, onde a vida se dá por meio de imposições que retiram a autonomia do ser:

Em primeiro lugar, todos os aspectos da vida são realizados no mesmo local. Em segundo lugar, cada fase da atividade diária do participante é realizada na companhia de um grupo ... todas elas tratadas da mesma forma e obrigadas a fazer as mesmas coisas em conjunto. Em terceiro lugar, todas as atividades diárias são rigorosamente estabelecidas em horários ... e toda a sequência de atividades é imposta de cima, por um sistema de regras formais explicitas e um grupo de funcionários. Finalmente, as várias atividades obrigatórias são reunidas num plano racional único ... (GOFFMAN, 2010, p.17-18)

O hospital psiquiátrico, enquanto instituição total, é encarregado de asilar pessoas que apresentam algum "distúrbio social". Por meio de regras totalitárias, o hospital exerce três funções principais sob esse grupo de indivíduos: os isolar da sociedade, punir o comportamento desviante, vigiar e controlar. O paciente é objeto de uma carreira moral, ou seja, "uma carreira composta pelas progressivas mudanças que ocorrem nas crenças que tem a seu respeito e a respeito dos outros que são significativos para ele" (GOFFMAN, 2010, p. 25).

Esses pressupostos refletem, como demanda e resultado, na ambientação das instituições totais. A estratégia de organização espacial para o controle e dominância é chamada de Disciplina (FOUCAULT, 1987, p. 127). A arquitetura foi vista como elemento inicial para conduzir, de forma racional (eficiência), as ações dos indivíduos e alcançar eficácia e efetividade nas realizações de atividades. A diferenciação em layout setorial refletia a diferenciação organizacional por classes de grupos de pessoas. Consequentemente, se estabelecia um rebatimento de posição hierárquica associado às circunstâncias de demanda do fazer. Guardadas as proporções, se buscam 
que os corpos adquiram movimentos, ora controlados, ora limitados pelas possibilidades físico-espaciais previamente arquitetadas. $O$ Panóptico é o exemplo irradiante dessa concepção. A estratégia clássica de vigilância é resolvida mediante a proposição de um modelo espacial-projetivo, onde os corpos subjugados se localizam no perímetro do anel em torno de uma torre de observação. Com essa ambientação, é fácil a vigia. O inspecionado, pela lógica da construção, tem a sensível ciência de permanente vigilância (FOCAULT, 1975, p.181). Desta forma, pode-se dizer que as instituições utilizam tal modelo por sua hierarquia entre ocupantes, mediante um poder de acesso à visibilidade. Como exemplo, tem-se o Hospital Colônia de Barbacena (fig.1)

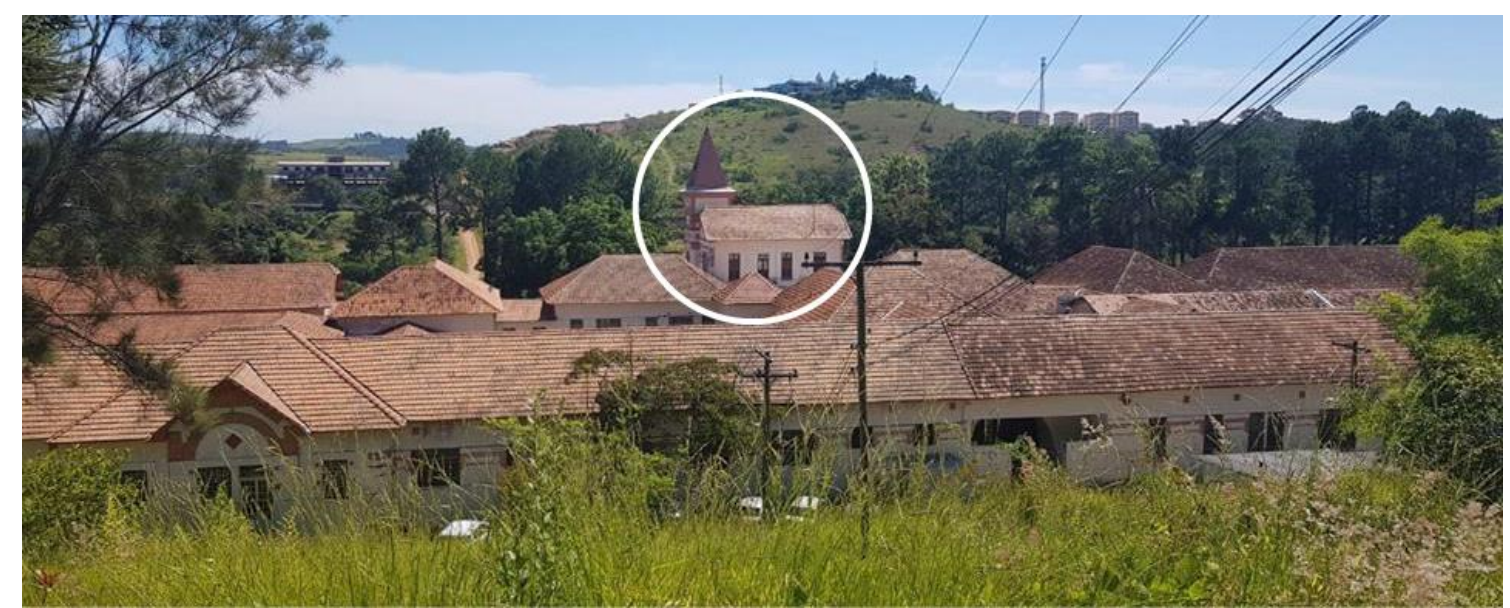

Fig.1: Torre de controle da Assistência de Alienados de Barbacena (atual FHEMIG). Foto dos autores.

Basaglia (1985), também considera o hospital psiquiátrico como uma "instituição difusa". Os pressupostos da instituição total, ou seja, a isolação, punição e controle e vigilância é transmito socialmente através de práticas e representações de conteúdos excludentes que contribuem para construir e/ou reforçar estereótipos e discriminações em relação ao usuário identificado como doente mental. Essa difusão virulenta, na sua dimensão sócio-afetiva, contamina todo o circuito institucional hospitalocêntrico, desde a atenção em saúde mental num ambulatório até o hospital, além de se estender para a sociedade como um todo. Os ex-pacientes ao receberem alta, ainda carregam o estigma de "violentos e incapazes", o que determinará o espaço social frio, controlado e de reclusão que irão ocupar mesmo estando no mundo externo (FILHO, NÓBREGA, 2010).

Nessa perspectiva, a Reforma Psiquiátrica pressupõe ser um processo de desconstrução da rede de instituições totais em Saúde Mental e das representações sociais excludentes por meio de estratégias políticas, sociais e técnico-profissionais. Também implica em construir equipamentos e novas práticas que questionam as formas recicladas da psiquiatria clássica, o que envolve as filosofias de tratamento, a forma de se relacionar com o portador de sofrimento mental e a maneira de conceber espaços para a terapia psíquica. As mudanças, portanto, são estruturadas de forma socio-histórica, comprometidas com liberdade e inclusão social, tendo como porta de entrada as organizações de equipamentos terapêuticos territoriais. Conjuntamente, esses tópicos envolvem um importante ponto de investigação arquitetônica, dado que trazem demandas e necessidades próprias ao 
desenvolvimento do espaço edificado para a Saúde Mental dentro do contexto da Reforma Psiquiátrica Brasileira.

\subsection{Reforma Psiquiátrica Brasileira e o CAPS}

Foi na segunda metade da década de 70 que culminou a Reforma Psiquiátrica Brasileira (RPB). Ela tem como principal objetivo a desinstitucionalização e a reabilitação psicossocial dos portadores de deficiência mental. Três processos foram fundamentais para que ela ocorresse: (1) ampliação dos atores e movimentos socias em busca da defesa da cidadania desses indivíduos, destacando o Movimento dos Trabalhadores em Saúde Mental (MTSM); (2) reformulação legislativa que impediu a contratação de novos hospitais psiquiátricos e proibição de internações compulsórias com o projeto de lei 3.657/89 (BRASIL, 1988); (3) surgimento de experiências institucionais bem-sucedidas em um novo tipo de cuidados em rede em Saúde Mental, como o CAPS Prof. Luiz da Rocha Cerqueira, em São Paulo e a intervenção na Casa de Saúde Anchieta, em Santos (SP) (TENÓRIO, 2002, p. 34). Todas essas reformulações preconizam superar os parâmetros totalitários manicomiais e considerar o usuário estruturante de uma "clínica ampliada", que o articula em meio à sua territorialidade para trata-lo.

As portarias 189/91 e 224/92 norteiam o modelo assistencial em uma rede de serviços extra-hospitalares e territorializados, que são: Serviços Residenciais Terapêuticos (SRTs), Núcleos de Atenção Psicossocial (NAPS), Centros de Atenção Psicossocial (CAPS) e unidades assistenciais da urgência e emergências. A organização desses novos serviços busca seguir os princípios de universalidade, hierarquização, regionalização e integralidade (BRASIL, 1988).

Segundo a Portaria no 336/2002 do Ministério da Saúde, os CAPS são os mais representativos desses serviços, que têm como prioridade "o atendimento de pacientes com transtornos mentais severos e persistentes em sua área territorial, em regime de tratamento intensivo, semi-intensivo e não intensivo" (BRASIL, 2002). A relação com o território é uma questão central para o serviço e aparece enunciada em diversos documentos como, na Portaria $n^{\circ} 336 / 02$ "[um CAPS vai] responsabilizar-se, sob coordenação do gestor local, pela organização da demanda e da rede de cuidados em saúde mental no âmbito do seu território" e, no Artigo 10. $\$ 2^{0}$, , eles"... deverão constituir-se em serviço ambulatorial de atenção diária que funcione segundo a lógica do território" (MINISTÉRIO DA SAÚDE, 2002). O CAPS é dividido em três modalidades (CAPS 1, CAPS II e CAPS III) de serviços, as quais cumprem a mesma função de atendimento em saúde mental, se diferenciando por ordem crescente de porte/complexidade e abrangência populacional. Além dos cuidados em saúde mental, o CAPS tem responsabilidade de supervisionar e capacitar equipes de atenção básica, serviços e programas no âmbito do seu módulo territorial (MINISTÉRIO DA SAÚDE, BRASIL, 2002).

\subsection{Territorialidade e o Atendimento Psicossocial}

O conceito de território permite uma visão conjunta dos diversos processos sociais, minimamente sócio técnicos, econômicos e políticos que ocorre no espaço. De acordo com Filho e Nóbrega (2004), o território pode ser considerado um objeto dinâmico, vivo, repleto de inter-relações e que possui influência recíproca entre sociedade e a vida do indivíduo no corpo social. 
Outro aspecto é que ele pode ser visto como um produto socioespacial das contradições da tríade economia, política e cultura (EPC). Além disso, o território está em permanente movimento de construção, desconstrução e reconstrução (FILHO, NÓBREGA, 2010 apud SAQUET, 2003). Gilles Deleuze (1989, p. 4) afirma que:

\begin{abstract}
o território só vale em relação a um movimento através do qual dele se sai" e "não há território sem um vetor de saída do território, e não há saída do território, ou seja, desterritorialização, sem, ao mesmo tempo, um esforço para se reterritorializar em outra parte (DELEUZE, 1989, p. 595).
\end{abstract}

Para Filho e Nóbrega (2010) apud Kastrup (2001, p.215), a territorialização nada mais é do que um processo de "habitar um território". Esta ação está associada a vinculação ao lugar que se refere ao sentimento de posse e afeições de pertencimento que o indivíduo desenvolve em relação a um território específico. De acordo com Soczka (2005), a vinculação ao lugar é constituída pelos laços experimentados como positivos em relação ao ambiente através de ligações comportamentais, afetivas e cognitivas estabelecidas entre os indivíduos e o espaço sócio/físico ao longo do tempo. O self do sujeito constitui uma conexão com o território que o circunda. No centro desta associação, situa-se o passado e o futuro ambiental da pessoa. $O$ passado ambiental refere-se as memórias associadas a um local, enquanto o futuro ambiental refere-se as expectativas a esse mesmo lugar baseado nas experiencias passadas. Estas cognições integram ideias, sentimentos, atitudes, valores, e preferências relacionadas com a complexidade dos contextos físicos da existência cotidiana. A vinculação ao lugar conduz a uma subestrutura da auto-identidadede que é compota por sentimentos de pertencimento, autonomia, segurança, satisfação e estabilidade acerca do ambiente (SOCZA, 2005).

A territorialização, desterritorialização e reterritorialização é uma marca da experiencia do portador de sofrimento mental quando é desopitalizado. Ao ser internado no manicômio, o sujeito deixa seu território e desfaz tudo aquilo que constitui como dimensão do familiar e do próprio para ingressar em uma instituição total. No hospital psiquiátrico, sofre ataques do seu EU para enquadrá-lo numa razão do Outro (objetificá-lo e dociliza-lo) de forma que o torne submisso as regras instituição, controlado pelo corpo médico e integrante de uma massa social obediente. Ao receber alta, há um nova percepção e ingresso ao meio coletivo: há uma recepção fria e hostil em que a liberação significa passar do "topo de um pequeno mundo para o ponto mais baixo de um mundo grande" (GOFFMAN, 2010, p.69). Goffman (2010, p.69) atribui o termo "estigma" para esse processo, onde a posição social do ex-paciente nunca mais será igual à que era antes da internação. Assim, o sujeito não retorna ao território o qual constituía o seu mundo de origem, ele precisará se reterritorializar na busca de construção de um novo mundo e um lugar para si.

Os equipamentos de Saúde Mental da Reforma Psiquiátrica, ao que tudo indica, possuem também a incumbência de cuidar e investir no processo de reterritorialização para produzir ações que facilite a reconstituição da sociabilidade, emancipação e integração social de seus usuários. A apropriação da cidade pelo ex-interno transborda o circuito de acesso aos serviços e redes de assistência e os espaços urbanos tornam-se lócus de 
sociabilidade. Organizar um serviço que opere segundo a lógica do território supõe o envolvimento dos contextos micro e macro social e os componentes culturais que circunda o sujeito. Elementos como a co-presença, a vizinhança, a intimidade, a cooperação e a socialização permitem o desenvolvimento de um tratamento voltado a pessoalidade. Para tanto, é preciso criar uma intensa porosidade entre o serviço e os recursos do seu entorno para potencializar a participação social e abrir lacunas ao espaço público como lócus terapêutico.

\section{METODOLOGIA}

O presente artigo é fruto de uma pesquisa de mestrado em desenvolvimento que é um estudo investigatório qualitativo sobre territorialidades dos portadores de sofrimento mental pós Reforma Psiquiátrica em Barbacena e Juiz de Fora, Minas Gerais. As populações de investigação serão usuários da rede de assistência à Saúde Mental de ambas as cidades. A amostra será constituída por moradores das Residências Terapêuticas que foram desospitalizados de hospitais psiquiátricos e de custódia de cada município. Será analisado o cotidiano desses usuários na utilização dos Centro de Atenção Psicossocial (CAPS), Unidade Básica de Saúde (UBS), ou outro dispositivo que auxilia no projeto terapêutico individual e a inserção destes moradores na rede social da comunidade (trabalho, lazer, educação, entre outros). O objetivo é entender como a ambiência extra-muros hospitalares traz uma nova dinâmica de vida para os sujeitos portadores de doenças mentais e como estes constroem novos territorialidades, contra os anteriores paradigmas e filosofias do sistema manicomial.

A estrutura do estudo tem uma abordagem histórico-temporal relativa à relação entre as terapias psiquiátricas de Pinel até a Reforma Psiquiátrica Brasileira. Além disto, serão utilizadas uma linha de investigação in loco, por meio de visitação as SRT e CAPS. Para a pesquisa de campo, procurar-se-á o aprofundamento de uma realidade específica na qual o sujeito observado está cotidianamente imerso e se caracteriza pelas investigações em que, além da pesquisa bibliográfica e/ou documental, se realizará também coletas de dados junto a estas pessoas, com o recurso de diferentes tipos de pesquisa.

Englobar-se-á também conceitos e técnicas da Psicologia Ambiental. Ela é um campo de conhecimento voltado para o estudo das relações recíprocas entre a pessoa-ambiente. A análise ambiental dentro deste campo disciplinar abrange três componentes: os físicos (arquitetura, equipamentos, objetos, etc.); os nãos físicos (aspectos psicológicos) e 3) os sociais. Estas dimensões compõem um todo a ser analisado, de modo que ao estudar qualquer um de seus componentes, deve-se levar em consideração os demais elementos do sistema ambiental (CAVALCANTE, ELALI; 2009).

A Abordagem Ecológica do Desenvolvimento de Bronfenbrenner (1996) servirá de contextualização para compreensão da análise ambiental com meio no qual o sujeito interage com seu ambiente. Bronfenbrenner explicita a necessidade de os pesquisadores estarem atentos para a diversidade que caracteriza o homem: seus processos psicológicos, sua participação dinâmica nos espaços, suas características pessoais e sua construção histórico-sóciocultural (ALVEZ, 1997). O indivíduo escolhido neste tipo de observação está dentro do seu próprio sistema, que engloba: o Microssistema: ambiente onde o 
indivíduo estabelece relações face-a-face; o Mesosistema: inclui inter-relações entre dois ou mais ambientes nos quais a pessoa participa ativamente; 0 Exossitema: ambientes onde a pessoa não se encontra presente, mas cujas relações que neles existem afetam seu cotidiano; o Macrossitema: abrange os sistemas de valores, crenças e culturas que são vivenciados pelo sujeito observado (BRONFENBRENNER, 1996). Salienta-se que no planejamento de investigação, tem-se foco no mesossistema, já mencionado.

A técnica de coleta de dados será divido em três partes: (1) Análise Descritiva: detalhar aspectos técnicos do ambiente arquitetônicos e urbanísticos, na forma física geométrico-geográfica e ambiental técnico locacional, tanto da Residência Terapêutica, CAPS e dos territórios dos sujeitos das observações; (2) Análise da AVD: investigação dos usuários e suas relações cotidianas com o objetivo de entender as diferenças entre o sistema manicomial e a rede assistencial de Saúde Mental no campo e caracterização do espaço-lugarambiência para o ser que ali vivencia (sentimentos e experimentações); (3) Análise de Territorialização: estudo da inclusão e construção de uma reterritorialização com a desospitalização que ocorreu com a nova política da Reforma Psiquiátrica, buscando entender o contexto social da pessoa neste processo (as etapas estão detalhadas na metodologia em anexo).

Após organizados as informações e dados coletados, será analisado como o território intervém na reabilitação psicossocial dos portadores de transtorno mental na cidade. A análise de dados será baseada na Pesquisa Fenomenológia (CRESWELL, 2014). Este tipo de estudo relata "o significado de uma experiencia vivida de vários indivíduos de um determinado fenômeno e descreve tais experiencias em uma essência universal" (CRESWELL, 2014, p.72). $\mathrm{O}$ investigador coleta os dados relativos aos cenários e ambientes vivenciados pelos sujeitos, analisa as informações, declarações e citações significativas e as combina em grupos de temas. Depois, o pesquisador desenvolve uma descrição textual das experiencias encontradas (o que as pessoas participantes experimentaram), uma descrição estrutural das suas experiencias (como elas as experimentaram em termos das condições, situações ou contextos) e as articula para transmitir uma essência geral da experiencia (CRESWELL, 2014, p.75). Na presente pesquisa, serão identificados expressões, vivências e sentimentos significativos do processo desospitalização e atividades de vida diária dos portadores de deficiência mental para demonstrar como esses aspectos estabelecem vínculos e identidades no território e os fenômenos que possam traduzir em pontos de alteração em relação ao campo teórico - histórico da vida em manicômios. Na síntese final do trabalho, será feito uma descrição narrativa, integrando os resultados dos dados levantados nas três etapas metodológicas e incluindo comentários dos participantes, do que é e como eles experimentam o processo da Reforma Psiquiátrica nas suas vidas.

\section{CONCLUSÃO}

A Reforma Psiquiátrica pressupõe um processo de desconstrução do aparato manicomial, o que inclui o hospital psiquiátrico, internações compulsórias e representações sociais excludentes, por meio de estratégias políticas, culturais e sociais. Estruturar equipamentos e novas práticas que auxilia a reterritorialização desses sujeitos na cidade é um elemento chave nesse contexto, o que envolve as ideologias terapêuticas, a maneira de conviver 
com as pessoas com sofrimento mental e a forma de projetar ambientes para o tratamento cognitivo. As transformações supõem ser estruturadas de forma socio-histórica, comprometidas com liberdade e inclusão social. A pesquisa que está sendo desenvolvida buscará entender as relações prático objetivas entre usuário, CAPS, SRTs e cidade em busca de analisar o processo de construção de novos territórios após a saída do hospital psiquiátrico. Isso envolve examinar a formação do sentimento de vinculação ao lugar pelo portador de transtorno mental, o que corresponde a interação dinâmica entre - meio social cotidiano (sobretudo entre amigos, família e vizinhos) e características do ambiente em que o mesmo acontece. O objetivo é observar se há a afeição de comunidade, que surge a partir do momento que a pessoa se percebe como pertencente a um grupo ou a um lugar específicos, entendendo que há uma relação única entre ambos. Os novos equipamentos que compõe a rede de assistência devem criar uma porosidade entre o serviço e comunidade para potencializar a inserção social e abrir brechas na cidade para ser local de reabilitação psicossocial. Espera-se que por meio dessa investigação possam ser encontrados fatores que demonstre que o CAPS coloca em prática as diretrizes da Reforma Psiquiátrica, articulando território, meio social e cultural para a reabilitação psicossocial das pessoas com sofrimento mental ou que aponte evidências que dificultam o processo de re-territorialização dessa população.

\section{REFERÊNCIAS}

ALVES, Paola Biasoli. A ecologia do desenvolvimento humano: experimentos naturais e planejados. Revista Scielo. Porto Alegre. v.10, n.2, 1997. Disponível em: <http://www.scielo.br/scielo.php? script=sci arttext\&pid=s010279721997000200013>. Acesso em: 08 de Abr. 2019.

AMARANTES, Paulo. Loucos pela vida: a trajetória da reforma psiquiátrica no Brasil. Rio de Janeiro: Editora Fio Cruz, 1995.

BRASIL, Ministério Da Saúde. Portaria № 336, de 19 de Fevereiro de 2002.

Disponível em:

<http://bvsms.saude.gov.br/bvs/saudelegis/gm/2002/prt0336 1902 2002.html>

BRASIL, Ministério da Saúde. Portaria № 3.090, de 23 de Dezembro de 2011. Acesso Disponível em:

<http://bvsms.saude.gov.br/bvs/saudelegis/gm/2011/prt309023 12 2011.html>

BRASIL, Ministério Da Saúde. Portaria Nº 336, de 19 de Fevereiro de 2002.

Disponível em:

<http://bvsms.saude.gov.br/bvs/saudelegis/gm/2002/prt033619 02 2002.html>

BRONFENBRENNER, Urie. A ecologia do desenvolvimento humano :

experimentos naturais e planejados. Editora Artes Medicas. Porto Alegre. 1996

CAVALCANTI, Sylvia; ELALI, G. (Orgs.). Temas básicos em psicologia

ambiental. Editora Vozes. Petrópolis, Rio de Janeiro. 2011.

DIPARTIMENTO DI SALUTE MENTALE TRIESTE. Triste: Storiadi um Cambiamento.

Trieste. Itália. 2019. Disponível em:

<http://www.triestesalutementale.it/storia/index.htm>. Acesso em: 11 de fev. 2019. 
FONTES, Maria Paula Zambrano. Imagens da arquitetura da saúde mental: Um estudo sobre a requalificação dos espaços da casa do sol - Instituto municipal de assistência à saúde Nise da Silveira. Dissertação de Mestrado. Programa de Pós-graduação em Arquitetura - Faculdade de Arquitetura e Urbanismo.

Universidade Federal do Rio de Janeiro. Rio de Janeiro. 2003.

FOUCAULT, Michel. Vigiar e Punir - História da Violência nas Prisões. Petrópolis, Vozes, 1987.

G1 Zona da Mata._Juiz de Fora é classificada como polo de saúde mental pelo governo federal. Disponível em: <http://gl globo.com/mg/zona-damata/noticia/2015/02/juiz-de-fora-e-classificada-como-polo-de-saude-mentalpelo-governo-federal.html>. Acesso em: 13 de maio de 2019.

GERDATH, Tatiana Engel; SILVEIRA, Denise Tolfo, 2009. Métodos de Pesquisa.

Editora UFRGS. 1 edição. Porto Alegre. Rio Grande do Sul. 2009. Disponível em: http://www.ufrgs.br/cursopgdr/downloadsSerie/derad005.pdf. Último acesso:

17 de maio de 2019.

LYNCH, K. A imagem da cidade. Tradução: Jefferson Luiz Camargo. São Paulo: Martins fontes, 1997.

MARTINS, Edna; SZYMANSKI, Heloisa. A abordagem ecológica de Urie

Bronfenbrenner em estudos com famílias. Revista Estudos e Pesquisas em

Psicologia. Rio de Janeiro. v.2004, n.1, 2004. Disponível em:

$<$ http://pepsic.bvsalud.org/scielo.php? script=sci arttext\&pid=S1808-

42812004000100006>. Acesso em: 08 de Abr. 2019.

PESSOTI, Isaias. O século dos manicômios. São Paulo: Editora 34, 1996.

RHEINGANTZ, Paulo Afonso; BRASILEIRO Alice; ALCANTARA Denise de; AZEVEDO Giselle Arteiro; QUEIROZ Mônica. Observando a qualidade do lugar:

procedimentos para a avaliação pós-ocupação. Coleção PROARQ/FAU/UFRJ. Rio de Janeiro. 2009

SILVA, Flávia Denise Barbosa Vasques. Relatório das atividades desenvolvidas pela Coordenação de Saúde Mental COSAM/SESAPS. Coordenação de Saúde Mental. Prefeitura Municipal de Barbacena - Minas Gerais. 2016.

TENÓRIO, Fernando. A Reforma Psiquiátrica brasileira, da década de 1980 aos dia atuais: história e conceitos. Revista História, Ciências, Saúde. Rio de Janeiro. V. 9, N.1, P.25-59, Jan-Abr. 2002.

YASUI, Silvio. Conhecendo as origens da Reforma Psiquiátrica Brasileira: as experiências francesa e italiana. Rio de Janeiro. Editora Fiocruz. 2009

WICK, Livia; SCHUTZ, Benjamin. Architectures for psychiatrictreatment. EPFL Écolepolytechniqueféderale de Lausanne, 2011. 